\title{
On the existence of global solution of the system of equations of liquid movement in porous medium
}

\author{
Margarita Tokareva ${ }^{1,2, *}$ and Alexander Papin ${ }^{1}$ \\ ${ }^{1}$ Institute of Mathematics and Information Technology, Department of Differential Equations, 656049 Lenina, 61, Barnaul, Russia \\ ${ }^{2}$ Lavrentyev Institute of Hydrodynamics SB RAS, 630090 Lavrentieva, 15, Novosibirsk, Russia
}

\begin{abstract}
The initial-boundary value problem for the system of one-dimensional isothermal motion of viscous liquid in deformable viscous porous medium is considered. Local theorem of existence and uniqueness of problem is proved in case of compressible liquid. In case of incompressible liquid the theorem of global solvability in time is proved in Holder classes. A feature of the model of fluid filtration in a porous medium considered in this paper is the inclusion of the mobility of the solid skeleton and its poroelastic properties. The transition from Euler variables to Lagrangian variables is used in the proof of the theorems.
\end{abstract}

\section{Introduction}

Mathematical models of fluid filtration in a porous medium apply to a broad range of practical problems. The examples include, but are not limited to filtration near river dams, irrigation and drainage of agricultural fields, dynamics of hydraulic fracturing during oil and gas mining, methane extraction from coal and shale deposits, flow of magma in the earth's crust, etc. Parameters of these models strongly depend on the properties of fluids as well as the porous solid medium. Thus, a vast number of models are currently available. Majority of these models, however, make a simplifying assumption of a static solid porous skeleton, and treat the porosity as a given function. In this work, we try to relax this assumption to account for the mobility and poroelastic properties of a solid component. Models with a given porosity function of a solid component are based on a Muskat-Leverett filtering theory. S.N. Antontsev and V.N. Monakhov [1] developed the theory for a special case of two-phase motion of immiscible incompressible liquids in a non-deformable porous medium. A large number of papers are devoted to numerical studies (see, for example, [2]).

Tertzagi [3] was the first to develop models of poroelastic media that would take into account mobility of the skeleton and its poroelastic properties. He introduced the principle of effective stress, defined as the difference between total stress and the pressure of the liquid phase. This position reflects the fact that the liquid carries a part of the load. The relation between the deformation of the skeleton of the solid matrix and the fluid flow is of key importance here. Bio [4] further developed Tertsagi's theory: he introduced a joint deformation model of a fluidsaturated porous medium and established the theory of poroelasticity. Almost simultaneously and independently, Frenkel [5] developed a similar theory. Later, V.N.
Nikolayevsky, P.P. Zolotarev, and Kh.A. Rakhmatullin [6], [7], [8] proposed analogous models in their studies.

O.B. Bocharov [9], allowed the porosity to depend on the pressure, but no deformation of the porous skeleton was considered. V. V. Vedernikov and V. N. Nikolaevskii [10] proposed a two-phase filtration model in a deformable porous medium with a solid skeleton motion being described analogous to the Tertsagi principle and the modified Hooke linear law. The model was not justified. This was done later in [11], [12], where particular solutions were derived. O.B. Bocharov et all [13] derived the properties of the solutions for a degenerate case. All filtration models are very complex both from a theoretical point of view as well as in their application to specific problems. Only a handful of studies on deformable porous media models published to date included result justification. A mainstream research is based on the classical theory of filtration, and model justification is only examined for a limited number of specific cases. Strict mathematical results are only presented in a few papers exploring the existence and uniqueness of solutions of such problems. For example, A. M. Abourabia et all in [14], [15], [16] reduced the initial system of equations to a single equation of higher order by making a number of simplifying assumptions. M. Simpson et all [16] proved a local solvability of the Cauchy problem in S.L. Sobolev spaces. Y. Geng et all [14], [15], investigated solutions of the "simple wave" type. Numerical studies of such problems were carried out, for example, in [17].

\section{Problem Statement}

For each component of a two-phase medium (the $s$ skeleton and the $f$ liquid phase contained in it), the concepts of volumes of the solid skeleton $V_{s}$ and pores $V_{p}$

\footnotetext{
* Corresponding author: tma25@ mail.ru
} 
are introduced. Then the specific pore volume (porosity, fraction of the volume of the medium happen to the voids) can be stated as $\phi=\frac{V_{p}}{V_{t}}$, where the total volume is $V_{t}=V_{p}+V_{s}$.

Darcy flow, which describes the fluid velocity relative to the solid velocity, is defined as $\vec{q}_{D}=\phi\left(\vec{v}_{f}-\right.$ $\vec{v}_{s}$ ), where $\vec{v}_{f}, \vec{v}_{s}$ are velocities of fluid and porous skeleton respectively [18].

Mass conservation laws for liquid and solid phases in absence of phase transitions look like [19]

$$
\begin{gathered}
\frac{\partial\left(\rho_{f} \phi\right)}{\partial t}+\nabla \cdot\left(\rho_{f} \phi \vec{v}_{f}\right)=0 \\
\frac{\partial(1-\phi) \rho_{s}}{\partial t}+\nabla \cdot\left((1-\phi) \rho_{s} \vec{v}_{s}\right)=0
\end{gathered}
$$

where $t$ is time, $\rho_{f}$ is density of liquid, $\rho_{s}$ is density of solid phase, $\nabla=\left(\frac{\partial}{\partial x_{1}}, \frac{\partial}{\partial x_{2}}, \frac{\partial}{\partial x_{3}}\right)$ is gradient operator, $\left(x_{1}, x_{2}, x_{3}\right)$ are Eulerian coordinates.

Laws of conservation of mass can be wrote in terms of material derivative: $\left(\frac{d}{d t}=\frac{\partial}{\partial t}+\vec{v}_{s} \cdot \nabla\right)$. Then we obtain

$$
\begin{gathered}
\frac{d \rho_{f} \phi}{d t}=-\nabla \cdot\left(\rho_{f}\left(\vec{q}_{D}+\phi \vec{v}_{s}\right)\right), \\
\nabla \cdot \vec{v}_{s}=\frac{1}{1-\phi} \frac{d \phi}{d t} .
\end{gathered}
$$

By motion of fluid in deformable medium it is assumed that [19], [20]:

1. the deviator of stress tensor in liquid phase is neglected $\left(S_{f}=0\right)$, because the fluid viscosity is much lower than the skeleton shear viscosity.

2. the total stress tensor $\sigma$ is described via stress tensors of solid phase $\sigma_{s}$ and liquid phase $\sigma_{f}$ by the rule:

$\sigma=(1-\phi) \sigma_{s}+\phi \sigma_{f}=(1-\phi)\left(S_{s}-p_{s} I\right)-\phi p_{f} I$, and total pressure is described as $p_{\text {tot }}=(1-\phi) p_{s}+$ $\phi p_{f}$, where $\sigma_{s}, p_{s}$ are stress tensor and pressure of solid phase, $S_{S}=2 \eta \dot{\varepsilon}_{D}$ is deviator of stress tensor, $\dot{\varepsilon}_{D}=$ $\frac{1}{2}\left(\frac{\partial v_{S}}{\partial \vec{x}}+\left(\frac{\partial v_{S}}{\partial \vec{x}}\right)^{*}\right)$ is deviatoric strain rate tensor, $\eta$ is the skeleton shear viscosity, $\sigma_{f}, p_{f}$ are stress tensor and pressure of liquid phase.

Terzaghi's principle states that deformation of the solid matrix is determined by an effective stress defined as [3] $\sigma_{e}=\sigma+p_{f} I$. Then, for a fully saturated, lowporosity media, the effective dynamic pressure is $p_{e}=$ $p_{\text {tot }}-p_{f}[21]$.

Notice, that

$$
d \phi=\frac{d V_{p}}{V_{t}}-V_{p} \frac{d V_{t}}{V_{t}^{2}}=\frac{d V_{p}}{V_{t}}-\phi \frac{d V_{t}}{V_{t}} .
$$

If density of solid phase $\rho_{s}$ is constant, then $d V_{s}=0$ and $d V_{t}=d V_{p}$. From equation (1) we obtain

$$
d \phi=(1-\phi) \frac{d v_{t}}{v_{t}} .
$$

The assumption that porosity is function of effective pressure was used in work [22] $\phi=\phi\left(p_{e}\right)$, in particular: $\phi=\phi_{0} \exp \left\{-b p_{e}\right\}$. In approach, used in [22], bulk compressibility of two-phase medium $\beta_{t}$ is defined as relativity summary change of volume, responding on changing applied effective dynamic pressure: $\beta_{t}=$ $-\frac{1}{V_{t}}\left(\frac{\partial V_{t}}{\partial p_{e}}\right)$.
Equation (2) in this case can be written as $d \phi=$ $-(1-\phi) \beta_{t} d p_{e}$.

Volumetric compressibility is also function of porosity, for example [23]: $\beta_{t}(\phi)=\phi^{b} \beta_{\phi}$, where $\beta_{\phi}$ is the bulk compressibility, $b$ is positive constant $\beta_{\phi}=$ $-1 / V_{p}\left(\partial V_{p} / \partial p_{e}\right)$. Thus the temporal variation of the porosity owing to mechanical compaction can be written as [24]:

$$
\frac{1}{1-\phi} \frac{d \phi}{d t}=-\beta_{t}(\phi) \frac{d p_{e}}{d t}
$$

The constitutive creep law can be written as [25], [26]

$$
\frac{1}{1-\phi} \frac{d \phi}{d t}=-\frac{p_{e}}{\xi}
$$

where $\xi$ corresponds to a bulk viscosity. This formulation is analogous to a creep-controlled viscous compaction law used in studies dealing with magma transport in the Earth's mantle [27].

The bulk viscosity as rule depends on $\phi$, for example: $\xi(\phi)=\frac{\eta}{\phi^{m}}$, where $m$ is positive constant [28].

Thus the rheological law combining mechanical and viscous compaction can be expressed as [23], [25]

$$
\frac{1}{1-\phi} \frac{d \phi}{d t}=-\beta_{t}(\phi) \frac{d p_{e}}{d t}-\frac{p_{e}}{\xi(\phi)} \text {. }
$$

The conservation of momentum for the fluid can be stated as Darcy's law [18], [29]

$$
\vec{q}_{D}=-K \nabla\left(\frac{P_{e x}}{\rho_{f} g}\right),
$$

where $K$ is the hydraulic conductivity, $K=\left(k^{\prime} \rho_{f} g\right) / \mu$, $k^{\prime}, \mu$ are the permeability and the fluid dynamic viscosity, $g$ is the density of the mass forces $(\vec{g}=$ $(0,0,-g)), P_{e x}$ is the excess fluid pressure, defined as the difference between the fluid pressure and the hydrostatic pressure: $P_{e x}=p_{f}-p_{h}$. In this way, we have

$$
\vec{q}_{D}=-\frac{k^{\prime}}{\mu}\left(\nabla p_{f}+\rho_{f} \vec{g}\right) .
$$

In some cases coefficients $k^{\prime}, \beta_{t}, \xi$ could be determined in experiment another way. In particular they can be determined as: $\beta_{t}=\phi^{b} \beta_{\phi}, \xi=\eta / \phi^{m}, k^{\prime}=k \phi^{n}$, where $k$ is permability, $b=1 / 2, m \in[0,2], n=3$ [23].

The laws of momentum conservation for each of phase can be written [30]

$$
\begin{gathered}
\nabla \cdot\left(\phi \sigma_{f}\right)-\rho_{f} \phi \vec{g}+M=0, \\
\nabla \cdot\left((1-\phi) \sigma_{s}\right)-\rho_{s}(1-\phi) \vec{g}-M=0,
\end{gathered}
$$

where $M$ is interphase pulse exchange.

Adding these equations, we obtain the equation of conservation of momentum of the system "solid matrix pore fluid" [19], [20], [24], namely: the equation of incompressible deformation of the solid skeleton matrix taking into account the effect of the pore fluid pressure:

$$
\nabla \cdot \sigma+\rho_{\text {tot }} \vec{g}=0
$$

where $\rho_{\text {tot }}=(1-\phi) \rho_{s}+\phi \rho_{f}$ is average density of the compressible medium.

In expanded form, the previous equation can be written [30]

$$
\rho_{t o t} \vec{g}+\operatorname{div}\left((1-\phi) \eta\left(\frac{\partial \vec{v}_{s}}{\partial \vec{x}}+\left(\frac{\partial \vec{v}_{s}}{\partial \vec{x}}\right)^{*}\right)\right)-\nabla p_{t o t}=0
$$


In some applications, the force balance equation is written as [19], [30].

$$
-\nabla p_{\text {tot }}+\rho_{\text {tot }} \vec{g}=0
$$

Thus, the equations of model in the absence of phase transitions have the form [19], [20], [23], [30]:

$$
\begin{gathered}
\frac{\partial(1-\phi) \rho_{s}}{\partial t}+\operatorname{div}\left((1-\phi) \rho_{s} \vec{v}_{s}\right)=0 \\
\frac{\partial\left(\rho_{f} \phi\right)}{\partial t}+\operatorname{div}\left(\rho_{f} \phi \vec{v}_{f}\right)=0 \\
\phi\left(\vec{v}_{f}-\vec{v}_{s}\right)=-k(\phi)\left(\nabla p_{f}+\rho_{f} \vec{g}\right) \\
\nabla \cdot \vec{v}_{s}=-a_{1}(\phi) p_{e}-a_{2}(\phi)\left(\frac{\partial p_{e}}{\partial t}+\vec{v}_{s} \cdot \nabla p_{e}\right) \\
\nabla \cdot \sigma+\rho_{t o t} \vec{g}=0, \rho_{t o t}=\phi \rho_{f}+(1-\phi) \rho_{s} \\
p_{t o t}=\phi p_{f}+(1-\phi) p_{s} \\
p_{e}=(1-\phi)\left(p_{s}-p_{f}\right)
\end{gathered}
$$

This quasilinear composite type system describes the spatial unsteady isothermal motion of a compressible fluid in a viscoelastic medium. Here $k(\phi), a_{1}(\phi), a_{2}(\phi)$ are parameters of poroelastic medium.

Numerical studies of various initial-boundary value problems for the system of equations (3)-(7) were carried out in the works [20], [23], [31]. Questions of justification in these papers were not considered. In some particular cases, the issues of justifying this model are discussed in [13], [32], [33], [34]. The the main difference this work from [34] is the taking into account the compressibility of liquid and additional term in the equation of conservation of momentum of the system on the whole. Work [33] is the short announcement of results of this work.

The system of equations describing the onedimensional unsteady motion of a compressible fluid in a viscous porous medium in the domain $(x, t) \in Q_{T}=\Omega \times$ $(0, T), \Omega=(0,1)$, is as follows [20], [30]:

$$
\begin{gathered}
\frac{\partial(1-\phi) \rho_{s}}{\partial t}+\frac{\partial}{\partial x}\left((1-\phi) \rho_{s} v_{s}\right)=0, \\
\frac{\partial\left(\rho_{f} \phi\right)}{\partial t}+\frac{\partial}{\partial x}\left(\rho_{f} \phi v_{f}\right)=0, \\
\phi\left(v_{f}-v_{s}\right)=-k(\phi)\left(\frac{\partial p_{f}}{\partial x}-\rho_{f} g\right), \\
\frac{\partial v_{s}}{\partial x}=-\frac{1}{\xi(\phi)} p_{e}, \\
p_{e}=p_{t o t}-p_{f}, p_{t o t}=\phi p_{f}+(1-\phi) p_{s}, \\
\frac{\partial}{\partial x}\left(2 \eta(1-\phi) \frac{\partial v_{s}}{\partial x}\right)=\rho_{t o t} g+\frac{\partial p_{t o t}}{\partial x}, \\
\rho_{t o t}=\phi \rho_{f}+(1-\phi) \rho_{s} .
\end{gathered}
$$

The problem is written in the Eulerian coordinates $x, t$. The real density of the solid particles $\rho_{s}$ are assumed constant, and Clapeyron dependence is taken for fluid $p_{f}=R \rho_{f}, R=$ const $>0$. At the boundary of the region $\Omega$, the velocities of the phases $v_{s}, v_{f}$ are set, and at the initial moment of time the density $\rho_{f}^{0}(x)$ and porosity $\phi^{0}(x)$ are set.

The following conditions are considered for the system (8)- (11):

$$
\begin{gathered}
\left.v_{s}\right|_{x=0,1}=\left.v_{f}\right|_{x=0,1}=0,\left.\quad \rho_{f}\right|_{t=0}=\rho^{0}(x), \\
\left.\phi\right|_{t=0}=\phi^{0}(x) .
\end{gathered}
$$

Following [1], [34], [35], [36] we rewrite the system (8)(11) in Lagrangian variables:

$$
\begin{gathered}
\frac{\partial(1-\phi)}{\partial t}+(1-\phi)^{2} \frac{\partial v_{s}}{\partial x}=0, \\
\frac{\partial}{\partial t}\left(\rho_{f} \frac{\phi}{1-\phi}\right)+\frac{\partial}{\partial x}\left(\rho_{f} \phi\left(v_{f}-v_{s}\right)\right)=0,
\end{gathered}
$$

$$
\begin{gathered}
\phi\left(v_{s}-v_{f}\right)=k(\phi)\left((1-\phi) \frac{\partial p_{f}}{\partial x}-\rho_{f} g\right), \\
(1-\phi) \frac{\partial v_{s}}{\partial x}=-\frac{1}{\xi(\phi)} p_{e}, p_{e}=p_{t o t}-p_{f} \\
(1-\phi) \frac{\partial}{\partial x}\left((1-\phi)^{2} \frac{\partial v_{s}}{\partial x}\right)= \\
\rho_{t o t} g+(1-\phi) \frac{\partial p_{t o t}}{\partial x}
\end{gathered}
$$

We will represent the equation (17) as

$$
\frac{\partial}{\partial x}\left((1-\phi)^{2} \frac{\partial v_{s}}{\partial x}-p_{t o t}-h\right)=0, \quad \frac{\partial h}{\partial x}=\frac{\rho_{t o t} g}{1-\phi} .
$$

Consequently

$$
(1-\phi)^{2} \frac{\partial v_{s}}{\partial x}-p_{t o t}-h=p^{0}(t),
$$

where $p^{0}(t)$ is the some time dependent function.

Taking into account equation (16), we obtain a representation for $p_{\text {tot }}$ :

$$
\begin{aligned}
p_{\text {tot }}=\left(p^{0}(t)-\right. & \left.\int_{0}^{x} \frac{\rho_{\text {tot }} g}{1-\phi} d \xi+\frac{(1-\phi)}{\xi(\phi)} p_{f}\right)(1 \\
& \left.+\frac{(1-\phi)}{\xi(\phi)}\right)^{-1}
\end{aligned}
$$

We represent the equation (16) as

$$
\frac{\partial v_{s}}{\partial x}=-\frac{1}{(1-\phi) \xi(\phi)}\left(p_{t o t}-p_{f}\right) .
$$

After integrate this equation by $x$ from 0 to 1 , taking into account the submission for $p_{\text {tot }}$, we get

$$
\begin{gathered}
p^{0}=\left(\int _ { 0 } ^ { 1 } \left(\frac { 1 } { ( 1 - \phi ) ( \xi ( \phi ) + 1 - \phi ) } \left(p_{f}\left(\rho_{f}\right)+\right.\right.\right. \\
\left.\left.\left.+\int_{0}^{x} \frac{\rho_{t o t} g}{1-\phi} d \xi\right)\right) d x-v_{s}(1, t)+v_{s}(0, t)\right) . \\
\cdot\left(\int_{0}^{1} \frac{1}{(1-\phi)(\xi(\phi)+1-\phi)} d x\right)^{-1} \equiv P^{0}\left(\phi, \rho_{f}\right) .
\end{gathered}
$$

Converting the original system of equations we get system for finding porosity and density (using equation (14), taking into account equation (15) and using equation (13), taking into account equation (16):

$$
\begin{aligned}
& \quad \frac{\partial}{\partial t}\left(a(\phi) \rho_{f}\right)-\frac{\partial}{\partial x}\left(K(\phi) b\left(\rho_{f}\right) \frac{\partial \rho_{f}}{\partial x}-\right. \\
& \left.\frac{K(\phi)}{1-\phi} \rho_{f}^{2} g\right)=0, \\
& \frac{\partial G}{\partial t}=p_{f}\left(\rho_{f}\right)-p^{0}+\int_{0}^{x} \frac{\rho_{t o t} g}{1-\phi} d \xi,
\end{aligned}
$$

Where

$$
\begin{array}{r}
a(\phi)=\frac{\phi}{1-\phi}, \quad K(\phi)=k(\phi)(1-\phi), \\
b\left(\rho_{f}\right)=\rho_{f} \frac{\partial p_{f}\left(\rho_{f}\right)}{\partial \rho_{f}},
\end{array}
$$

and the function $G$ is defined as follows:

$$
\frac{\partial G}{\partial \phi}=1+\frac{\xi(\phi)}{1-\phi} \text {. }
$$

\section{Compressible Fluid} written as

For system (18), (19) conditions (12) could be

$$
\begin{aligned}
& \left.\left((1-\phi) \frac{\partial p_{f}\left(\rho_{f}\right)}{\partial x}-\rho_{f} g\right)\right|_{x=0, x=1}=0, \\
& \left.\rho_{f}\right|_{t=0}=\rho^{0}(x),\left.\phi\right|_{t=0}=\phi^{0}(x),
\end{aligned}
$$




$$
\begin{gathered}
p^{0}=\int_{0}^{1}\left(\frac { 1 } { ( 1 - \phi ) ( \xi ( \phi ) + 1 - \phi ) } \left(p_{f}\left(\rho_{f}\right)\right.\right. \\
\left.\left.+\int_{0}^{x} \frac{\rho_{\text {tot }} g}{1-\phi} d \xi\right)\right) d x \\
\cdot\left(\int_{0}^{1} \frac{1}{(1-\phi)(\xi(\phi)+1-\phi)} d x\right)^{-1} \equiv P^{0}\left(\phi, \rho_{f}\right) .
\end{gathered}
$$

In the notation of function spaces we follow [37]: $C^{k+\alpha, m+\beta}\left(Q_{T}\right)-$ Hölder's space, where $k, m$ are natural numbers, $(\alpha, \beta) \in(0,1]$, with the norm $\|f\|_{C^{k+\alpha, m+\beta}\left(Q_{T}\right)}$.

The solution of problem (18)-(20) is the set of functions $\phi, \rho_{f} \in C^{2+\alpha, 1+\beta}\left(Q_{T}\right)$, such that $0<\phi<$ $1, \rho_{f}>0$. These functions satisfy the equations (18)(19) and the initial and boundary conditions (20) and regarded as continuous functions in $Q_{T}$.

Theorem 1. Suppose that the data of problem (18)-(20) satisfies the following conditions:

1. the functions $k(\phi), \xi(\phi)$ and their derivatives up to the second order are continuous for $\phi \in(0,1), \rho_{f}>0$, and satisfy the conditions $k_{0}^{-1} \phi^{q_{1}}(1-\phi)^{q_{2}} \leq k(\phi) \leq$ $k_{0} \phi^{q_{3}}(1-\phi)^{q_{4}}, 1 / \xi(\phi)=a_{0}(\phi) \phi^{\alpha_{1}}(1-\phi)^{\alpha_{2}-1}, 0<$ $R_{1} \leq a_{0}(\phi) \leq R_{2}$, where $k_{0}, \alpha_{i}, R_{i}, i=1,2$ are positive constants, $q_{1}, \ldots, q_{4}$ are fixed real parameters,

2. the initial functions $\phi^{0}, \rho^{0}$ and function $g$ satisfy the following smoothness conditions: $\phi^{0} \in C^{2+\alpha}(\bar{\Omega}), \rho^{0} \in$ $C^{2+\alpha}(\bar{\Omega}), g \in C^{1+\alpha, 1+\alpha / 2}\left(\bar{Q}_{T}\right), \quad$ and the matching conditions

$$
\left.\left(\left(1-\phi^{0}\right) \frac{d p_{f}\left(\rho^{0}\right)}{d x}-\rho^{0} g(x, 0)\right)\right|_{x=0, x=1}=0,
$$

as well as satisfy the inequalities $0<m_{0} \leq \phi^{0}(x) \leq$ $M_{0}<1,0<m_{1} \leq \rho^{0}(x) \leq M_{1}<\infty, 0<g(x, t) \leq$ $g_{0}<\infty, x \in \bar{\Omega}$, where $m_{0}, M_{0}, m_{1}, M_{1}, g_{0}$ are given positive constants.

Then problem (18)-(20) has a local solution, i.e., there exists a value of $t_{0} \in(0, T)$ such that $\left(\phi(x, t), \rho_{f}(x, t)\right) \in C^{2+\alpha, 1+\alpha / 2}\left(\bar{Q}_{t_{0}}\right)$. Moreover,

$$
0<\phi(x, t)<1, \rho_{f}(x, t)>0 \text { в } \bar{Q}_{t_{0}} .
$$

The solvability of problem (18)-(20) is established by using the Tikhonov-Schauder Fixed-Point Theorem [1].

Since the function $\psi=G(\phi)$ is strictly monotone, at $\phi \in(0,1)$, that the inverse function is exist: $\phi=$ $G^{-1}(\psi)$. Assuming that $\rho(x, t)=\rho_{f}(x, t)-$ $\rho^{0}(x), \quad \omega(x, t)=G(\phi)-G\left(\phi^{0}\right)$. We represent the equations (18), (19) in the form

$$
\begin{gathered}
\frac{\partial}{\partial t}\left(a(\omega)\left(\rho+\rho^{0}\right)\right)= \\
\frac{\partial}{\partial x}\left(K(\omega) b\left(\rho+\rho^{0}\right) \frac{\partial\left(\rho+\rho^{0}\right)}{\partial x}-\frac{K(\omega)}{1-\phi(\omega)}(\rho+\right. \\
\left.\left.\rho^{0}\right)^{2} g\right), \\
\frac{\partial \omega}{\partial t}=p_{f}\left(\rho+\rho^{0}\right)-p^{0}+\int_{0}^{x} \frac{\rho_{t o t} g}{1-\phi(\omega)} d \xi \\
\text { Here } a(\omega)=\frac{\phi(\omega)}{1-\phi(\omega)}, K(\omega)=k(\phi(\omega))(1- \\
\phi(\omega)), \phi(\omega)=G^{-1}\left(\omega+G\left(\phi^{0}\right)\right) . \text { Moreover, } \\
\left.\rho\right|_{t=0}=\omega_{t=0}=
\end{gathered}
$$

$$
\left.\left((1-\phi(\omega)) \frac{\partial\left(\rho+\rho^{0}\right)}{\partial x}-\left(\rho+\rho^{0}\right) g\right)\right|_{x=0, x=1}=0 \text {. }
$$

For the Banach space, we choose the space $C^{2+\beta, 1+\beta / 2}\left(\bar{Q}_{t_{0}}\right)$, where $\beta$ is any number from the interval $(0, \alpha), \alpha \in[0,1)$. Let

$$
\begin{gathered}
V=\left\{\bar{\rho}(x, t), \bar{\omega}(x, t) \in C^{2+\alpha, 1+\alpha / 2}\left(\bar{Q}_{t_{0}}\right) \mid\right. \\
\left.\bar{\rho}\right|_{t=0}=\left.\bar{\omega}\right|_{t=0}= \\
\left.\left((1-\phi(\bar{\omega})) \frac{\partial\left(\bar{\rho}+\rho^{0}\right)}{\partial x}-\left(\bar{\rho}+\rho^{0}\right) g\right)\right|_{x=0, x=1}=0, \\
\widehat{m}_{1}-\rho^{0}(x) \leq \bar{\rho}(x, t) \leq \widehat{M}_{1}-\rho^{0}(x)<\infty, \\
\widehat{m}_{1}=\frac{m_{1}}{2}\left(1+\frac{g_{0}}{R\left(1-M_{0}\right)}\right)^{-1}, \\
\widehat{M}_{1}=2 M_{1}\left(1+\frac{g_{0}}{R\left(1-M_{0}\right)}\right), \\
G\left(\frac{m_{0}}{2}\right)-G\left(\phi^{0}\right) \leq \bar{\omega}(x, t) \leq G\left(\frac{M_{0}+1}{2}\right)-G\left(\phi^{0}\right) \\
<\infty, \quad(x, t) \in Q_{t_{0}}, \\
\left(|\bar{\omega}|_{1+\alpha,(1+\alpha) / 2, Q_{t_{0}}},|\bar{\rho}|_{1+\alpha,(1+\alpha) / 2, Q_{t_{0}}}\right) \leq K_{1}, \\
\left(|\bar{\omega}|_{\left.2+\alpha,(2+\alpha) / 2, Q_{t_{0}},|\bar{\rho}|_{2+\alpha,(2+\alpha) / 2, Q_{t_{0}}} \leq K_{1}+K_{2}\right\}}\right.
\end{gathered}
$$

where $K_{1}$ is an arbitrary positive constant, while the positive constant $K_{2}$ will be given later. We note that on the set $V$ following inequalities hold: $0<m_{0} / 2 \leq$ $\phi(\bar{\omega}) \leq\left(M_{0}+1\right) / 2<1, \quad a(\bar{\omega})>0, \quad K(\bar{\omega})>0$.

Let us construct an operator $\Lambda$ mapping $V$ in $V$. Suppose that $\bar{\omega}, \bar{\rho} \in V$. Using (22), we define the function $\omega$ by the equality

$$
\begin{gathered}
\omega=\int_{0}^{t}\left(R\left(\bar{\rho}(x, \tau)+\rho^{0}(x)\right)-P^{0}(\phi(\bar{\omega}), \bar{\rho})+\right. \\
\left.\int_{0}^{x} g\left(\rho_{s}+\left(\bar{\rho}+\rho^{0}(\xi)\right) \frac{\phi(\bar{\omega})}{1-\phi(\bar{\omega})}\right) d \xi\right) d \tau .
\end{gathered}
$$

From the representation (23) it follows that smoothness $\omega$ is determined by the smoothness of functions $\bar{\rho}, \bar{\omega}, \rho^{0}, p^{0}$ and $g$. Therefor there exists a value $t_{1}=$ $t_{1}\left(m_{0}, M_{0}, m_{1}, M_{1}\right)$, such that for all $t_{0} \leq t_{1}$ the following inequality holds

$$
0<\frac{m_{0}}{2} \leq \phi(x, t) \leq \frac{M_{0}+1}{2}, \quad(x, t) \in Q_{t_{0}} .
$$

In particular, we have an estimate

$|\omega|_{2+\alpha, 1+\alpha / 2, Q_{t_{0}}}$

$\leq C_{0}\left(m_{0}, M_{0}, m_{1}, M_{1}, K_{1}, T,|g|_{1+\alpha, \Omega},\left|\rho^{0}\right|_{2+\alpha, \Omega},\left|p^{0}\right|_{\alpha / 2,[0, T]}\right)$ $\cdot\left(1+t_{0}\left|\bar{\rho}_{x x}\right|_{\alpha, \alpha / 2, \Omega}\right)$.

Taking into account (24) we also have the estimate for function $\omega(x, t)$ :

$$
G\left(\frac{m_{0}}{2}\right) \leq \omega(x, t)+G\left(\phi^{0}\right) \leq G\left(\frac{M_{0}+1}{2}\right) .
$$

Using (21), $\bar{\rho}$ and $\bar{\omega}(x, t)$, we find the function $\rho(x, t)$ as a solution of the problem (here and elsewhere, we assume that the initial and boundary conditions are matched):

$$
\begin{gathered}
\frac{\partial}{\partial t}\left(a(\bar{\omega})\left(\rho+\rho^{0}\right)\right)= \\
\frac{\partial}{\partial x}\left(K(\bar{\omega}) b(\bar{\rho}) \frac{\partial\left(\rho+\rho^{0}\right)}{\partial x}-\frac{K(\bar{\omega})}{1-\phi(\bar{\omega})}\left(\bar{\rho}+\rho^{0}\right)\left(\rho+\rho^{0}\right) g\right),
\end{gathered}
$$

$\left.\rho\right|_{t=0}=0$,

$$
\left.\left((1-\phi(\bar{\omega})) R \frac{\partial\left(\rho+\rho^{0}\right)}{\partial x}-\left(\rho+\rho^{0}\right) g\right)\right|_{x=0, x=1}=0 .
$$

The equation for $\rho(x, t)$ is uniformly parabolic. In view of the properties of $\bar{\omega}(x, t)$ and $\rho^{0}(x)$ the problem (25) has a classical solution [37]. In addition, we have the following estimate: 


$$
\left|\frac{1}{a(\bar{\omega})} \frac{\partial a(\bar{\omega})}{\partial t}\right| \leq C_{1}\left(m_{0}, M_{0}, m_{1}, M_{1}, \max _{0 \leq t \leq T}\left|p^{0}(t)\right|\right) .
$$

Under the additional condition smallness for the value of the time interval the following statement holds.

Lemma 1. There exists such a $t_{2}$, that when $t_{0} \leq$ $\min \left(t_{1}, t_{2}\right)$, the classical solution of problem (25) satisfies the following inequality in $\mathrm{Q}_{\mathrm{t}_{0}}: 0<\widehat{\mathrm{m}}_{1} \leq$ $\rho(\mathrm{x}, \mathrm{t})+\rho^{0}(\mathrm{x}) \leq \widehat{\mathrm{M}}_{1}<\infty$.

The proof fully follows [33].

In view of Lemma 1 and the properties of $\bar{\omega}$, we have the following estimates [37]:

$$
\begin{gathered}
|\rho|_{\alpha, \alpha / 2, Q_{t_{0}}} \leq C_{2},|\rho|_{2+\alpha, 1+\frac{\alpha}{2}, Q_{t_{0}}} \leq \\
\leq C_{3}\left(1+\left|\rho^{0}\right|_{2+\alpha, \Omega}+\left|\bar{\rho}_{x}\right|_{\alpha, \alpha / 2, Q_{t_{0}}}+\left|\bar{\omega}_{t}\right|_{\alpha, \alpha / 2, Q_{t_{0}}}\right. \\
\left.+\left|\bar{\omega}_{x}\right|_{\alpha, \alpha / 2, Q_{t_{0}}}\right)
\end{gathered}
$$

in which the constants $C_{2}, C_{3}$ depend on $K_{1}, m_{0}, m_{1}, M_{0}$, $M_{1}$. Therefore $\quad|\rho|_{2+\alpha, 1+\alpha / 2, Q_{t_{0}}} \leq$ $C_{4}\left(K_{1}, m_{0}, m_{1}, M_{0}, M_{1}\right)$. Let $C_{5}=\max \left\{C_{0}, C_{4}\right\}$. Choose $K_{2}$ so that $C_{5} \leq\left(K_{1}+K_{2}\right) / 2$. Then, for $t_{0}<$ $\min \left(t_{1}, t_{2},\left(K_{1}+K_{2}\right)^{-1}\right)$ we obtain $|\rho|_{2+\alpha, 1+\alpha / 2, Q_{t_{0}}} \leq$ $K_{1}+K_{2}, \quad|\omega|_{2+\alpha, 1+\alpha / 2, Q_{t_{0}}} \leq K_{1}+K_{2}$. It remains to verify conditions $\quad|\rho|_{1+\alpha,(1+\alpha) / 2, Q_{t_{0}} \leq}$ $K_{1}, \quad|\omega|_{1+\alpha,(1+\alpha) / 2, Q_{t_{0}}} \leq K_{1}$. Integrating equation (25) with respect to time, we obtain $|\rho|_{0, Q_{t_{0}}} \leq C_{6} t_{0}$. From the equation (23) we obtain $|\omega|_{0, Q_{t_{0}}} \leq C_{7} t_{0}$. Further, using for $\rho, \omega$ an inequality of the form [1, p.35]

$|u|_{1+\alpha,(1+\alpha) / 2, Q_{t_{0}}} \leq C|u|_{2+\alpha, 1+\alpha / 2, Q_{t_{0}}}^{c}|u|_{0, Q_{t_{0}}}^{1-c} \quad c=$ $(1+\alpha)(2+\alpha)^{-1}$,

we find that there exists a sufficiently small value of $t_{0}$, depending on $K_{1}$ and $K_{2}$, such that the required estimates hold: $|\rho|_{1+\alpha,(1+\alpha) / 2, Q_{t_{0}}} \leq K_{1}, \quad|\omega|_{1+\alpha,(1+\alpha) / 2, Q_{t_{0}}} \leq K_{1}$. Thus, the operator $\Lambda$ maps the set $V$ into itself for sufficiently small values of $t_{0}$. Using the estimates obtained above, we can easily show the continuity of the operator $\Lambda$ in the norm of the space $C^{2+\beta, 1+\beta / 2}\left(\bar{Q}_{t_{0}}\right)$. By the Tikhonov-Schauder theorem, there exists a fixed point $(\rho, \omega) \in V$ of the operator $\Lambda$. Uniqueness is established in the standard way [32]. Theorem 1 is proved.

\section{The Case of Incompressible Medium}

If the density of the liquid phase is constant $\left(\rho_{f}=\right.$ const) the system of equations (18), (19) can be reduced to one equation for the porosity of $\phi$ in Lagrange variables [34]:

$\left.\left.\left.\rho_{f}\right)\right)\right)$.

$$
\frac{\partial}{\partial t}\left(\frac{\phi}{1-\phi}\right)=\frac{\partial}{\partial x}\left(k ( \phi ) \left((1-\phi) \frac{\partial^{2} G(\phi)}{\partial x \partial t}-g\left(\rho_{t o t}+\right.\right.\right.
$$

This equation is supplemented by the following initialboundary conditions:

$$
\begin{gathered}
\left.\phi\right|_{t=0}=\phi^{0},\left(k ( \phi ) \left((1-\phi) \frac{\partial^{2} G(\phi)}{\partial x \partial t}-\right.\right. \\
\left.\left.g\left(\rho_{t o t}+\rho_{f}\right)\right)\right)\left.\right|_{x=0,1}=0 .
\end{gathered}
$$

Definition 2. The solution of problem (28), (29) is the function $\phi, \phi_{t} \in C^{2+\alpha, 1+\beta}\left(Q_{T}\right)$, such that $0<\phi<1$. This functions satisfy the equation (28) and the initial and boundary conditions (29) and regarded as continuous functions in $Q_{T}$.

Theorem 2. Suppose that the data of problem (28), (29) satisfies the following conditions:

1) the functions $k(\phi), \xi(\phi)$ and their derivatives up to the second order are continuous for $\phi \in(0,1)$ and satisfy the conditions $k_{0}^{-1} \phi^{q_{1}}(1-\phi)^{q_{2}} \leq k(\phi) \leq$ $k_{0} \phi^{q_{3}}(1-\phi)^{q_{4}}, 1 / \xi(\phi)=a_{0}(\phi) \phi^{\alpha_{1}}(1-$

$\phi)^{\alpha_{2}-1}, \quad 0<R_{1} \leq a_{0}(\phi) \leq R_{2}<\infty$, where $k_{0}, \alpha_{i}, R_{i}, i=1,2$ are positive constants, $q_{1}, \ldots, q_{4}$ are fixed real parameters.

2) the function $g$ and the initial function $\phi^{0}$ satisfy the following smoothness conditions: $g \in$ $C^{1+\alpha, 1+\beta}\left(\bar{Q}_{T}\right), \quad \phi^{0} \in C^{2+\alpha}\left(\bar{\Omega}_{T}\right)$, and inequalities $0<$ $m_{0} \leq \phi^{0}(x) \leq M_{0}<1, \quad|g(x, t)| \leq g_{0}<\infty, \quad x \in \bar{\Omega}$, where $m_{0}, M_{0}, g_{0}$ are given positive constants.

Then problem (28)-(29) has a local solution, i.e., there exists a value of $t_{0}$ such that $\phi(x, t) \in C^{2+\alpha, 1+\beta}\left(\bar{Q}_{t_{0}}\right)$. Moreover $0<\phi(x, t)<1$ in $\bar{Q}_{t_{0}}$.

Theorem 3. Let, in addition to the conditions of Theorem 2, the functions $k(\phi), \xi(\phi)$ satisfy the conditions $\quad k(\phi)=(k / \mu) \phi^{n}, \quad \xi(\phi)=\eta \phi^{-m}, n \geq$ $1, m \geq 1$, where $k, \mu, \eta$ are given positive constants. Then for all $t \in[0, T], T<\infty$ uniqueness solution of problem (28), (29) exists, and there are numbers $0<$ $m_{1}<M_{1}<1$ such that $m_{1} \leq \phi(x, t) \leq M_{1}, \quad(x, t) \in$ $Q_{T}$.

\section{Local solvability}

Putting $z(x, t)=\partial G / \partial t$, we proceed from (28) and (29) to the following problem for $G$ and $z$

$$
\begin{gathered}
z=\frac{\partial G}{\partial t},\left.\quad G\right|_{t=0}=G\left(\phi^{0}\right) \equiv G^{0}(x), \\
\frac{z}{d(G)}-\frac{\partial}{\partial x}\left(a(G) \frac{\partial z}{\partial x}-b(G)\right)=0, \\
\left.\left(a(G) \frac{\partial z}{\partial x}-b(G)\right)\right|_{x=0, x=1}=0,
\end{gathered}
$$

where $\quad d(G)=(1-\phi(G)) \xi(\phi(G)), \quad a(G)=$ $k(\phi(G))(1-\phi(G)), \quad b(G)=k(\phi(G)) g((1-$ $\left.\phi(G)) \rho_{s}+(1+\phi(G)) \rho_{f}\right)$.

Solvability in small is established by the Tikhonov-Schauder Fixed-Point Theorem [1].

Let $\omega(x, t)=G(\phi)-G\left(\phi^{0}\right)$. We represent the equations (30), (31) as

$$
\begin{gathered}
z=\frac{\partial \omega}{\partial t},\left.\quad \omega\right|_{t=0}=0, \\
\frac{z}{d(\omega)}-\frac{\partial}{\partial x}\left(a(\omega) \frac{\partial z}{\partial x}-b(\omega)\right)=0, \\
\left.\quad\left(a(\omega) \frac{\partial z}{\partial x}-b(\omega)\right)\right|_{x=0, x=1}=0 .
\end{gathered}
$$

As a Banach space, we choose the space $C^{2+\zeta, 1+\gamma}\left(\bar{Q}_{t_{0}}\right)$, where $\zeta$ is any number from the interval $(0, \alpha), \alpha \in[0,1), \gamma$ is any number from the interval $(0, \beta), \beta \in[0,1)$. Let

$$
\begin{array}{r}
V=\left\{\bar{\omega} \in C^{2+\alpha, 1+\beta}\left(\bar{Q}_{t_{0}}\right)|\bar{\omega}|_{t=0}=0,0<\frac{m_{0}}{2} \leq \phi(\bar{\omega})\right. \\
\leq \frac{M_{0}+1}{2}<\infty, \\
\left.|\bar{\omega}|_{1+\alpha,(1+2 \beta) / 2, Q_{t_{0}}} \leq K_{1},|\bar{\omega}|_{2+\alpha, 1+\beta, Q_{T_{0}}} \leq K_{1}+K_{2}\right\},
\end{array}
$$


where $K_{1}$ is positive constant, and positive constant $K_{2}$ will be specified later. We construct an operator $\Lambda$ mapping $V$ in $V$ in the same way as in [34] and we have following estimates:

$$
\begin{gathered}
|z|_{2+\alpha, \Omega} \leq N_{1}\left(K_{1}, m_{0}, M_{0}\right), \quad|z|_{2+\alpha, \beta, Q_{t_{0}}} \\
\leq N_{2}\left(K_{1}, m_{0}, M_{1}\right) . \\
|\omega|_{2+\alpha, 1+\beta, Q_{t_{0}}} \leq N_{3}\left(K_{1}, m_{0}, M_{0}\right)\left(1+t\left|z_{x x}\right|_{\alpha, \beta, Q_{t_{0}}}\right) \\
|\omega|_{0, Q_{t_{0}}} \leq N_{4} t_{0}, \quad|\omega|_{1+\alpha,(1+2 \beta) / 2, Q_{t_{0}}} \leq K_{1} .
\end{gathered}
$$

Let $N_{5}=\max \left\{N_{1}, N_{3}\right\}$. We choose $K_{2}$ so that $N_{5} \leq$ $\left(K_{1}+K_{2}\right) / 2$. Then for $t_{0}=2\left(K_{1}+K_{2}\right)^{-1}$ we obtain $|\omega|_{2+\alpha, 1+\beta, Q_{t_{0}}} \leq K_{1}+K_{2}$.

Based on the estimates for $\omega$, the property $0<$ $\frac{m_{0}}{2} \leq \phi(x, t) \leq \frac{M_{0}+1}{2}$ can be easily obtained as follows. Let $0<\phi_{1} \leq \phi_{2}<1$. From the definition of the functions $G(\phi)$ and $\xi(\phi)$ we have

$$
\begin{gathered}
0<\Delta G \equiv G\left(\phi_{2}\right)-G\left(\phi_{1}\right)= \\
\int_{\phi_{1}}^{\phi_{2}}\left(1+\frac{\xi(s)}{1-s} d s\right) \geq\left(1+\frac{1}{R_{2}}\right)\left(\phi_{2}-\phi_{1}\right) .
\end{gathered}
$$

We have an estimate of the form $\left(1+R_{2}\right) \mid G\left(\phi_{1}\right)-$ $G\left(\phi_{2}\right)|\geq| \phi_{1}-\phi_{2} \mid$. In this way, we have estimate

$$
\begin{aligned}
& \left|\phi(x, t)-\phi^{0}(x)\right| \leq \delta(t), \\
& \delta(t) \longrightarrow 0 \text { при } t \longrightarrow 0,
\end{aligned}
$$

which implies, that there exists a value $t_{3}=t_{3}\left(m_{0}, M_{0}\right)$, such that for all $t_{0} \leq t_{3}$ the following inequality holds

$$
0<\frac{m_{0}}{2} \leq \phi(x, t) \leq \frac{M_{0}+1}{2}, \quad(x, t) \in Q_{t_{0}} .
$$

Thus, the operator $\Lambda$ maps the set $V$ into itself for sufficiently small values of $t_{0}$. Using the estimates obtained above, we can easily show the continuity of the operator $\Lambda$ in the norm of the space $C^{2+\zeta, 1+\gamma}\left(\bar{Q}_{t_{0}}\right)$. According to the Tikhonov-Schauder theorem, there exists a fixed point $\omega \in V$ of the operator $\Lambda$. Uniqueness of the solution of problem (30)-(32) is establish in the same way as in [34]. Theorem 2 is proved.

\section{Global solvability}

Proof of the theorem 3. By virtue of Theorem 2, we assume that a solution to the problem (28)-(29) exists on the interval $\left[0, t_{0}\right]$, with $0<\phi(x, t)<1, x \in \Omega, t \in$ $\left[0, t_{0}\right]$. After obtaining the necessary a priori estimates that are independent of the value of $t_{0}$, the local solution can be extended to the entire segment $[0, T]$.

Let $s(x, t)=\phi /(1-\phi)$ in (28) Then the function $s$ satisfies the next problem

$$
\begin{aligned}
& \frac{\partial s}{\partial t}-\frac{\partial}{\partial x}\left(\bar{k}(s)\left(\frac{1}{1+s} \frac{\partial^{2} G(s)}{\partial x \partial t}-\bar{g}(\phi(s))\right)\right)=0, \\
\left.s\right|_{t=0}= & s^{0}, \\
& \left.\left(\frac{1}{1+s} \frac{\partial^{2} G(s)}{\partial x \partial t}-\bar{g}(\phi(s))\right)\right|_{x=0, x=1}=0,
\end{aligned}
$$

where

$$
\begin{gathered}
\bar{k}(s)=k(\phi(s)), \\
\bar{g}(s)=g\left(\frac{1}{1+s} \rho_{s}+\frac{1+2 s}{1+s} \rho_{f}\right), s^{0}=\frac{\phi^{0}}{1-\phi^{0}} .
\end{gathered}
$$

The following Lemma holds.
Lemma 2. Let $s(x, t)$ is the solution of the problem (37), (38). Then $\int_{0}^{1} s(x, t) d x=\int_{0}^{1} s^{0}(x) d x \equiv \lambda$, and there is such a point $a(t) \in[0,1]$, that $s(a(t), t)=\lambda>0$.

The proof fully follows [1].

Further consider the function $\psi(s)$, that satisfies the relation

$$
\frac{d^{2} \psi(s)}{d s^{2}}=\bar{k}^{-1}(1+s) \frac{d G(s)}{d s} .
$$

Lemma 3. Let $s(x, t)$ is the solution of the problem (37), (38), $n, m \geq 1$. Then function $\psi$ satisfies the following inequality $\psi \geq P \frac{(1+s)^{2}}{2}$, where $P=\frac{\mu \eta}{k}=$ const $>0$.

For the function $G$ we have for $s>0$

$$
\begin{aligned}
& \frac{d G}{d s}=1+\eta s^{-m}(1+s)^{m-1}=1+\eta\left(1+\frac{1}{s}\right)^{m-1} \frac{1}{s} \\
& \geq \eta \frac{1}{s} .
\end{aligned}
$$

Then

$$
\frac{d^{2} \psi}{d s^{2}}=\bar{k}^{-1}(s)(1+s) \frac{d G}{d s}=\bar{P} s\left(\frac{1+s}{s}\right)^{\bar{p}}+P\left(\frac{1+s}{s}\right)^{p},
$$$$
\text { where } \bar{p}=n+1 \geq 2, p=m+n \geq 2, \bar{P}=\frac{\mu}{k} \text {. }
$$

If $\bar{p}, p$ are integers numbers, then function $\psi$ satisfy equality

$$
\begin{aligned}
& \psi=\bar{P}\left(\frac{\bar{p} s^{2}}{2}+s\left(c_{3}-\frac{\bar{p}(\bar{p}-1)}{2}\right)+c_{4}+\frac{\bar{p}(\bar{p}-1)}{2} \bar{L}\right. \\
& \left.+\frac{s^{3}}{6}+\sum_{i=1}^{\bar{p}-3} C_{p}^{i+3} \frac{1}{i+1} \frac{1}{s^{i}}\right)+ \\
& +P\left(\begin{array}{c}
\frac{s^{2}}{2}+s\left(c_{1}-p\right)+c_{2}+p L \\
+\sum_{i=3}^{p} C_{p}^{i} \frac{1}{(i-1)(i-2)} \frac{1}{s^{i-2}}
\end{array}\right), C_{p}^{i}=\frac{p !}{i !(p-i) !} .
\end{aligned}
$$

where $c_{1}, c_{2}, c_{3}, c_{4}$ are arbitrary constants,

$$
\bar{L} \equiv\left(s-\frac{(\bar{p}-2)}{3}\right) \ln s, \quad L \equiv\left(s-\frac{(p-1)}{2}\right) \ln s .
$$

It is enough to check the non-negativity of functions $\bar{L}, L$. Since

$$
\begin{gathered}
(\bar{L})_{s}^{\prime}=1+\ln s-\frac{p-2}{3 s},(\bar{L})^{\prime \prime}{ }_{s}=\frac{1}{s^{2}}\left(s+\frac{p-2}{3}\right)>0, \\
(L)_{s}^{\prime}=1+\ln s-\frac{p-1}{2 s}, \quad(L)^{\prime \prime}{ }_{s}=\frac{1}{s^{2}}\left(s+\frac{p-1}{2}\right)>0,
\end{gathered}
$$

then functions $\bar{L}, L$ are concave functions.

The minimum points of functions $\bar{s}^{*}, s^{*}$ satisfy, respectively, the inequalities

$$
1+\ln \bar{s}^{*}=\frac{p-2}{3 \bar{s}^{*}}, 1+\ln s^{*}=\frac{p-1}{2 s^{*}} .
$$

Let us show the boundedness of the functions $\bar{s}^{*}, s^{*}$. Since

$$
\bar{s}^{*}\left(1+\ln \bar{s}^{*}\right)=\frac{\bar{p}-2}{3}>0, s^{*}\left(1+\ln s^{*}\right)=\frac{p-1}{2}>0,
$$

then

$$
\bar{s}^{*}\left(1+\ln \bar{s}^{*}\right)>0, s^{*}\left(1+\ln s^{*}\right)>0 .
$$

Therefor $\bar{s}^{*}>e^{-1}, s^{*}>e^{-1}$. If $\bar{s}^{*}, s^{*} \geq 1$, then from equality

$$
\bar{s}^{*}\left(1+\ln \bar{s}^{*}\right)=\frac{\bar{p}-2}{3}>0, s^{*}\left(1+\ln s^{*}\right)=\frac{p-1}{2}>0
$$

follows

$$
1+\ln \bar{s}^{*} \leq \frac{\bar{p}-2}{3}, 1+\ln s^{*} \leq \frac{p-1}{2} .
$$


Hence

$$
e^{-1}<\bar{s}^{*} \leq e^{\frac{p-2}{3}}, e^{-1}<s^{*} \leq e^{\frac{p-3}{2}} .
$$

Choosing constants $c_{1}, c_{2}, c_{3}, c_{4}$ as follows:

$$
\begin{gathered}
c_{2} \geq \frac{p}{s^{*}}\left(s^{*}-\frac{p-1}{2}\right)^{2}+\frac{1}{2}, c_{1} \geq p+1, c_{3} \geq \frac{\bar{p}(\bar{p}+1)}{2}, \\
c_{4} \geq \frac{\bar{p}}{2}\left(1+\left|\frac{\bar{p}(\bar{p}-1)(\bar{p}-2)}{6 \bar{s}^{*}}\left(\bar{s}^{*}-\frac{p-2}{3}\right)\right|\right),
\end{gathered}
$$

we obtain

$$
\psi \geq \hat{P} \frac{(1+s)^{2}}{2}>0, \quad \hat{P}=\min \{\bar{P}, P\} .
$$

If $\bar{p}, p$ are not integers, then we take $\bar{p}=\bar{r}+\bar{q}, p=r+$ $q$, where $\bar{r}, r$ are integers parts, and $\bar{q}, q$ are fractional parts. Then function $\psi$ satisfy the equation

$$
\frac{d^{2} \psi}{d s^{2}}=P\left(1+\frac{1}{s}\right)^{r}\left(1+\frac{1}{s}\right)^{q}+\bar{P} s\left(1+\frac{1}{s}\right)^{\bar{r}}\left(1+\frac{1}{s}\right)^{\bar{q}} .
$$

Let introduce a function $\bar{\psi}$, satisfying equality

$$
\frac{d^{2} \bar{\psi}}{d s^{2}}=P\left(1+\frac{1}{s}\right)^{r}+\bar{P} s\left(1+\frac{1}{s}\right)^{\bar{r}} .
$$

Let us show that the difference $\psi-\bar{\psi}$ is positive. We have

$$
\frac{d^{2}(\psi-\bar{\psi})}{d s^{2}}=\delta(s) \geq 0 .
$$

Let $\varepsilon>0$. In the case $0<\varepsilon \leq s$ we represent the previous equality in the form

$$
\frac{d^{2}}{d s^{2}}\left(\psi-\bar{\psi}-\int_{\varepsilon}^{s}\left(\int_{\varepsilon}^{\xi} \delta(\tau) d \tau\right) d \xi\right)=0 .
$$

Whence it follows that the difference $\psi-\bar{\psi}$ satisfies the condition

$$
\begin{gathered}
\psi-\bar{\psi}=\int_{\varepsilon}^{s}\left(\int_{\varepsilon}^{\xi} \delta(\tau) d \tau\right) d \xi+c_{3}+c_{4} s \geq c_{3}+c_{4} s, \\
0<\varepsilon \leq s,
\end{gathered}
$$

where $c_{3}, c_{4}$ are arbitrary constants.

If $s<\varepsilon$, we represent the equation for the difference $\psi$ and $\bar{\psi}$ in the form

$$
\frac{d^{2}}{d s^{2}}\left(\psi-\bar{\psi}-\int_{s}^{\varepsilon}\left(\int_{s}^{\xi} \delta(\tau) d \tau\right) d \xi\right)=0 .
$$

This yields $\psi-\bar{\psi} \geq c_{3}+c_{4} s, \quad 0<s<\varepsilon$.

Finally, choosing constants $c_{3}, c_{4}>0$, we have $\psi-\bar{\psi}>$ 0 .

Lemma 3 is proved.

We give an example of the function $\psi$. Let $m=n=$ $\eta=k=\mu=1$. Then

$$
\psi=(2 s-1) \ln s+\frac{s^{2}}{2}+s+\frac{3}{2} \geq \frac{(1+s)^{2}}{2} .
$$

Lemma 4. Let $s(x, t)$ is the solution of the problem (37), (38) $n, m \geq 1$. Then there exists positive constants $A$ and $B$ such that $0<A \leq s \leq B<\infty$ for all $t \in[0, T]$, where

$$
\begin{gathered}
A=\lambda e^{-1 / \eta N_{6}^{1 / 2}}, B=\lambda e^{1 / \eta N_{6}^{1 / 2}}, \\
N_{6}=\int_{0}^{1}\left(\psi\left(s^{0}\right)+G_{x}^{2}\left(s^{0}\right)\right) d x \exp \left\{T N_{7}\right\}, \\
N_{7}=\frac{1}{2} \max \left\{1, \frac{4 g_{0}^{2}}{P}\left(\rho_{s}^{2}+4 \rho_{f}^{2}\right)\right\} .
\end{gathered}
$$

The proof of Lemma 4 and all subsequent reasoning fully follows [34]. Then we obtain Hölder estimates for $x$ and $t$ [34]. Further we use the theory of elliptic equations for the function $z$ set forth in [37]. Theorem 3 is proved.

\section{Conclusion}

In this article, we described the system of equations of one-dimensional movement of a viscous liquid in a deformable viscous porous medium. We proved the local solvability of this problem in case of compressible liquid and global solvability in Holder classes in case of incompressible liquid. In future we want to take into account dependences pressure of temperature and density.

The work of the first author was supported by the Russian Science Foundation (Project No. 19-11-00069).

\section{References}

1. S. N. Antontsev, A. V. Kazhikhov, V. N. Monakhov, Boundary-Value Problems of the Mechanics of Inhomogeneous Fluids. (Novosibirsk: Nauka, 1983) (in Russian)

2. A. N. Konovalov, Proc. of the Steklov Institute of Mathematics, 122, 3-23 (1973) (In Russian)

3. K. Terzaghi Theoretical Soil Mechanics. (New York: Jhon Wiley, 1943)

4. M. A. Biot, J. Appl. Phys., 12, 155-164 (1941)

5. J. I. Frenkel, Izv. Acad. Nauk USSR, 8 (1944)

6. V. V. Nikolaevskiy, Engineering Journal, 3 (1963)

7. P. P. Zolotarev, Engineering Journal, 4 (1964)

8. H. A. Rahmatulin, Applied Mathematics and Mechanics, 10 (1956)

9. O. B. Bocharov, Dinamika Sploshn. Sredy: Sb. nauch. tr., 50, 15-36 (1981) (in Russian)

10. V. V. Vedernikov, V. N. Nikolaevsii, Izvestiya Akademii Nauk SSSR, Mekhanika Zhidkosti i Gaza, 5, 769-773 (1978)

11. O. B. Bocharov, V. Ya. Rudyak, A. V. Seryakov, Journal of mining science, 50, (2014).

12. O. B. Bocharov, V. Ya. Rudyak, A. V. Seryakov, Hierarchical sequence of models and deformation peculiarities of porous media saturated with fluids, in Proceedings of the XLI Summer SchoolConference Advanced Problems in Mechanics (APM-2013), 183-190 (2013)

13. M. A. Tokareva, Journal of Siberian Federal University. Mathematics \& Physics, 8(4), 467-477 (2015)

14. A. M. Abourabia, K. M. Hassan, A. M. Morad, Chaos Solutions Fract 42, 1170-1180 (2009)

15. Y. Geng, L. Zhang, Applied Mathematics and computation, 217, 1741-1748 (2010)

16. G. Simpson, M. Spiegelman, M. I. Weinstein, Nonlinearity, 20, 21-49 (2007) 
17. A. S. Saad, B. Saad, M. Saad, Computers and Mathematics with Applications, 71, 565-584 (2016)

18. J. Bear, Dynamics of Fluids in Porous Media. (Elsevier, New York, 1972)

19. O. Coussy, Poromechanics (John Wiley and Sons, Chichester, U.K., 2004)

20. C. Morency, R. S. Huismans, C. Beaumont, P. Fullsack, Journal of Geophysical Research, 112 (2007)

21. A. W. Scempton, Effective stress in soils, concrete and ricks. Proceeding of the Conference on Pore Pressure and Suction in soils, Butterworths, London, 4-16 (1960)

22. L. F. Athy, Amer. Ass. Petrol. Geol. Bull., 14, 1-24 (1930)

23. J. A. D. Connolly, Y. Y. Podladchikov, Geodin. Acta., 11, 55-84 (1998)

24. D. M. Audet, A. C. Fowler, Geophys. J. Int., 110, 577-590 (1992)

25. A. C. Fowler, J. Geophys. Res., 104, 12,989-12,997 (1999)

26. F. Schneider, J. L. Potdevin, S. Wolf, I. Faille, Tectonophysics, 263, 307-317

27. D. P. McKenzie, J. Petrol, 25, 713-765 (1984)

28. R. A. Birchwood, D. L. Turcotte, J. Geophys. Res., 99, 20,051--20,058 (1994)

29. B. S. Massey, Mechanics of fluids, 6th ed., (Chapman and Hall, Boston, Mass, 1989).

30. A. Fowler Mathematical Geoscience. (SpringerVerlag London Limited, 2011)

31. X. S. Yang, Nonlinear Proceeses in Geophysics, vol. 7, 1-7 (2000)

32. A. A. Papin, M. A. Tokareva, Journal of Physics: Conference Series, 894 (2017)

33. A. A. Papin, M. A. Tokareva, IOP Conf. Series: Journal of Physics: Conf. Series, 1268 (2019)

34. A. A. Papin, M. A. Tokareva, Journal of Applied and Industrial Mathematics, 13(2), 350-362 (2019)

35. A. A. Papin, I. G. Akhmerova, Mathematical Notes, 87, 230-243 (2010)

36. A. A. Papin, I. G. Akhmerova, Mathematical Notes, 96, 166-179 (2014)

37. O. A. Ladyzhenskaya, N. N. Ural'tseva Linear and Quasilinear Equations of Elliptic Type, (Moscow: Nauka, 1973). (in Russian) 